\title{
Clinical Features of COVID-19 in Children
}

\author{
(1) Zümrüt Şahbudak Bal, (1) Zafer Kurugöl, (1) Ferda Özkınay \\ Ege University Faculty of Medicine, Department of Pediatrics, İzmir, Turkey
}

\begin{abstract}
In early December, pneumonia cases of unknown origin started to appear and, on the $7^{\text {th }}$ of January 2020 , these cases were declared to be caused by a novel beta-coronavirus according to viral genome sequencing on the $11^{\text {th }}$ of February, 2020. Coronaviruses are enveloped, single strand RNA viruses that have been known to have the ability to mutate rapidly, alter tissue tropism and adjust to different epidemiological situations. As of the end of April 2020, 122,392 laboratory-confirmed cases of COVID-19 had been detected in Turkey, of whom 3,258 died. From the beginning of the COVID-19 epidemic, children seem to be less affected than adults. Therefore, there are limited data regarding the clinical features of COVID-19 in children. The majority of children with confirmed COVID-19 had a history of household contact. The most common symptoms were fever and cough. Previous data suggest that nearly half of patients are afebrile at the onset of the disease. Hospitalization and PICU admission rates for children were lower than for adults. However, PICU admission can be necessitated in children with severe disease. Infants, particularly under the age of 12 months, were more likely to develop severe disease. In children, milder and asymptomatic cases can be challenging and can play a role in transmission. In particular, clinicians should test those children who have a history of family cluster even though they are asymptomatic or present with mild symptoms.
\end{abstract}

Keywords: COVID-19, SARS-CoV-2, child, clinical feature

\section{Introduction}

In early December, pneumonia cases of unknown origin were reported from Wuhan, a city in the Hubei province of China (1). On the $7^{\text {th }}$ of January, 2020, these cases were declared to be caused by a novel beta-coronavirus according to viral genome sequencing and the World Health Organization (WHO) announced a new name for the epidemic disease caused by 2019-nCoV: COVID-19 which is the acronym of "coronavirus disease 2019" (2). The International Committee on Taxonomy of Viruses renamed the virus 'Severe Acute Respiratory syndrome coronavirus-2' (SARS-CoV-2) which was initially named '2019-nCoV' (3). On the $11^{\text {th }}$ of March 2020, WHO declared the outbreak as a pandemic (4). As of the end of April 2020, 122,392 cases of laboratory-confirmed COVID-19 had been detected in Turkey, of whom 3,258 died (5). The main difference between COVID-19 and seasonal influenza-associated pneumonia is the potential severity of disease even in young adults without comorbidities. Earlier in the pandemic, COVID-19 seemed to affect older patients and male patients more and children were less affected. However, it was later understood that SARS-CoV-2 can affect all age groups but clinical severity depends on advancing age. In this article, we aimed to summarize the clinical features of SARS-CoV-2 in children.

Coronaviruses are enveloped, single strand RNA viruses that are known to have the ability to mutate rapidly, alter tissue tropism and adjust to different epidemiological 
situations (6). Coronaviruses can lead to respiratory, enteric, hepatic, and neurologic diseases. SARS coronavirus (SARSCoV), Middle East Respiratory syndrome coronavirus (MERSCoV) and SARS-CoV-2 are members of beta-coronavirus es (7). SARS-CoV-2 is the $7^{\text {th }}$ known coronavirus which can infect humans (7). Four of these (229E, NL63, OC43 and HKU1) cause mild upper respiratory tract infections (common cold), while the SARS-CoV and the MERS-CoV have more severe outcomes such as atypical pneumonia or death. Full genome sequencing analysis of SARS-CoV-2 demonstrated $89 \%$ nucleotide identity with BAT SARSlike-COVZXC21 and 82\% nucleotide identity with SARS-CoV $(8,9)$. It is spread by human-to-human transmission via droplets or direct contact (9).

Based on the search of the previous English literature, there are few reports regarding COVID-19 in children (1018). According to published reports, the median age of cases varied between 1 and 11 years and male gender dominance was observed (1-18). A nationwide study in China demonstrated that all children are vulnerable to SARS-CoV-2 infection. The main clinical features of adult patients include fever, dry cough, dyspnea and myalgia. When compared with adults, children have milder clinical symptoms (11). Regarding the categories described by Dong et al. (11), asymptomatic, mild disease and moderate cases accounted for $98 \%$ of total cases. The remaining $2 \%$ of patients had severe or critical illness. They evaluated 728 proven and 1,407 suspected SARS-CoV-2 pediatric cases and showed younger infants (below 1 year of age) developed more severe clinical symptoms than children above 1 year of age. With regards to severely or critically ill infant cases (10.6\% of all infant cases), severely ill was defined as having pneumonia and central cyanosis (8.7\% of all infant cases) and critically ill was defined as developing Acute Respiratory Distress syndrome (ARDS) requiring mechanical ventilation (1.8\% of infant cases). Severely or critically ill cases accounted for $7.3 \%$ of cases for those aged 1 to 5 years and $4.2 \%$ of cases for those aged 6 to 15 years. There was no death (11).

Data from the United States supports this finding that children younger than 18 years of age who had Covid-19 composed only $1.7 \%$ of the total number of patients for whom age was known ( $n=149,082)$. The majority of these children had household contact history (12). Of the 291 children for whom symptoms were recorded; $56 \%$ of these children had fever, $54 \%$ had cough and $13 \%$ had shortness of breath. They found that $93 \%$ of adult patients had at least one of the following symptoms: fever, cough or shortness of breath. However, only $73 \%$ of children had one of these symptoms. In addition, myalgia, headache, vomiting and diarrhea were less frequently observed in children than in adults. Only $5.7 \%$ of children were hospitalized and the hospitalization rate was highest among infants aged less than 1 year. Intensive care unit admission was also less frequent than for adults. Of the 345 children for whom medical history could be obtained, 23\% had at least one underlying disease and the most common underlying disease was chronic pulmonary disease (40\%) followed by cardiovascular disease and immune suppression (12).

Another large series on children from China by Lu et al. (13), consisting of 171 proven cases, found that the most common symptom was cough followed by pharyngeal erythema and fever. Interestingly, they observed that pharyngeal erythema was more common than fever. The majority of these cases were between 6 and 10 years of age and presented most commonly with pneumonia (64.9\%). Nearly all patients $(90.1 \%)$ had a history of family cluster. They performed thorax computed tomography (CT) for 111 of these 171 patients. Regarding chest CT findings, ground glass opacity was the most common finding followed by local patchy shadowing and bilateral patchy shadowing, 32.7\%, $18.7 \%$ and $12.3 \%$, respectively. Three patients required Pediatric Intensive Care Unit (PICU) admission and 1 of them died due to intussusception and multiorgan failure 4 weeks after the onset of the disease.

Zheng et al. (14) reported on 25 proven cases of COVID19 with a median age of 3 years. The majority of these patients were below 3 years of age and the male $(n=14)$ to female $(n=11)$ ratio was $1.27: 1$. Twenty-one $(84 \%)$ had contact history while the remaining 4 patients did not have any epidemiological history. The most common symptoms were fever (52\%) and dry cough (44\%). Chest CT was performed in 24 of these 25 patients and they found most frequently bilateral involvement (48\%), followed by unilateral involvement (20\%). Two patients had an underlying disease and two patients required mechanical ventilation.

A recent study from Italy, a country which has been greatly affected by the pandemic, evaluated 100 pediatric patients with COVID-19 (15). One different finding was the lack of epidemiological history in 55\% of these cases, which was higher than previous studies. The authors explained this finding as being due to the late lockdown in Italy. Similar to previous studies, the most common finding was fever $(54 \%)$ followed by cough (44\%), feeding problems $(23 \%)$, rhinorrhea $(22 \%)$ and shortness of breath (11\%). Less frequent symptoms included nausea, vomiting, diarrhea, 
fatigue, sore throat and rash. COVID-19 was more likely to affect children younger than 21 months of age. None of the patients died.

The most recent study from Spain by Tagarro et al. (16) evaluated 365 children with suspected COVID-19 from whom SARS-CoV-2 pharyngeal swabs were obtained. Fortyone of these cases were positive. Twenty-five of them were hospitalized, 4 patients were admitted to PICU and 4 patients required respiratory support beyond nasal prongs. Only 1 patient had an underlying disease (recurrent wheezing). The most common clinical presentation was upper respiratory tract (34\%) followed by viral bronchiolitis/pneumonia (27\%), fever of unknown origin (27\%) and gastroenteritis/ vomiting (5\%). Mortality was not observed.

COVID-19 PICU surveillance data from the United States reported that 74 children who were classified as critical had required PICU admission as of the $6^{\text {th }}$ April, 2020 (17). They estimated that 118,887 children would be admitted to hospitals due to severe COVID-19, of whom 13,038 would develop critical disease and be admitted to PICU. They suggested that testing to determine positive cases can initiate epidemiologic case-containment efforts, therefore reducing household and child-to-child transmission. Kam et al. (18) reported an infant who had positive nasopharyngeal swabs until day 16 of admission and demonstrated a high viral load. The infant was widely asymptomatic with only 1 peak of fever $\left(38.5^{\circ} \mathrm{C}\right)$, which returned to normal level within 1 hour. He returned negative on day 18 of admission. This case emphasizes the fact that asymptomatic children can excrete the virus and so play a role in transmission. Another recent study evaluated 9 pediatric patients and their families and showed that the child's onset of disease was later when compared with their family and also the duration of positive stool PCR was longer than for adults (19).

\section{Conclusion}

As a conclusion, children seem to be less affected by the COVID-19 pandemic than adults but this difference is still an open question. The majority of children testing positive to COVID-19 had a history of family cluster and therefore COVID-19 seems to have been transmitted by a family member. Another important point is the removal of children from family members who have contacted the disease to decrease household-transmission. The most common symptoms were fever and cough. Previous data suggest that nearly half of patients are afebrile at the onset of the disease. Less frequent symptoms are fatigue, myalgia, vomiting/nausea and gastroenteritis. Hospitalization and PICU admission rates were lower for children than for adults. However, PICU admission may be necessitated in children with severe disease. Infants, particularly those under the age of 12 months, were more likely to develop severe disease. Although children more commonly have milder or asymptomatic disease, their duration of positive PCR can be longer than for adults, and therefore they can play a role in transmission. Milder clinical symptoms can also cause challenges in the early diagnosis and infection control measures. Clinicians should test children, in particular those having a family member with COVID-19, even though they are asymptomatic or present with mild symptoms.

\section{Ethics}

Peer-review: Internally peer-reviewed.

\section{Authorship Contributions}

Concept: Z.Ş.B., F.Ö., Z.K., Design: Z.Ş.B., F.Ö., Z.K., Data Collection or Processing: Z.Ş.B., F.Ö., Z.K., Analysis or Interpretation: Z.Ş.B., F.Ö., Z.K., Literature Search: Z.Ş.B., F.Ö., Z.K., Writing: Z.Ş.B.

Conflict of Interest: I and my partners have had no potential conflict of interest.

Financial Disclosure: I and my partners have had no relevant financial interests.

\section{References}

1. Zhu N, Zhang D, Wang W, et al. China Novel Coronavirus Investigating and Research Team. A Novel Coronavirus from Patients with Pneumonia in China, 2019. N Engl J Med 2020;382:727-33.

2. World Health Organization. WHO Director-general's remarks at the media briefing on 2019-nCoV on 11 February 2020, 2020. Available at: https://www.who.int/dg/speeches/detail/whodirector-general-s-remarks-at-the-media-briefing-on-2019ncov-on-11-february-2020. Accessed 11 February 2020.

3. Gorbalenya AE, Baker SC, Baric RS, et al. The species Severe acute respiratory syndrome-related coronavirus: classifying 2019nCoV and naming it SARS-CoV-2. Nat Microbiol 2020;5:536-44.

4. World Health Organization (WHO). WHO Director-General's opening remarks at the media briefing on COVID-19 - 11 March 2020. Geneva: 11 Mar 2020. Available from: https://www. who.int/dg/speeches/detail/who-director-general-s-openingremarks-at-the-media-briefing-on-covid-19---11-march-2020.

5. Türkiye Cumhuriyeti Sağlık Bakanlığı.https://covid19.saglik.gov. tr/Accessed at May1,2020.

6. Helmy YA, Fawzy M, Elaswad A, Sobieh A, Kenney SP, Shehata AA. The COVID-19 Pandemic: A Comprehensive Review of Taxonomy, Genetics, Epidemiology, Diagnosis, Treatment, and Control. J Clin Med 2020;9. 
7. Jin $\mathrm{Y}$, Yang H, Ji W, et al. Virology, Epidemiology, Pathogenesis, and Control of COVID-19. Viruses 2020;12.

8. Lu R, Zhao X, Li J, et al. Genomic characterisation and epidemiology of 2019 novel coronavirus: implications for virus origins and receptor binding. Lancet 2020;395:565-74.

9. Cevik M, Bamford C, Ho A. COVID-19 pandemic - A focused review for clinicians. Clin Microbiol Infect. 2020 Apr 25.

10. Bassetti M, Vena A, Giacobbe DR. The novel Chinese coronavirus (2019-nCoV) infections: Challenges for fighting the storm. Eur ) Clin Invest 2020;50:e13209.

11. Dong $\mathrm{Y}, \mathrm{Mo} \mathrm{X}, \mathrm{Hu}$ Y, et al. Epidemiology of COVID-19 Among Children in China. Pediatrics. 2020 Mar 16. pii: e20200702.

12. CDC COVID-19 Response Team. Coronavirus Disease 2019 in Children - United States, February 12-April 2, 2020. MMWR Morb Mortal Wkly Rep 2020;69:422-26.

13. Lu X, Zhang L, Du H, et al. Chinese Pediatric Novel Coronavirus Study Team. SARS-CoV-2 Infection in Children. N Engl I Med 2020 Mar 18

14. Zheng $\mathrm{F}$, Liao $\mathrm{C}$, Fan $\mathrm{QH}$, et al. Clinical Characteristics of Children with Coronavirus Disease 2019 in Hubei, China. Curr Med Sci 2020 Mar 24.
15. Parri N, Lenge M, Buonsenso D. Coronavirus Infection in Pediatric Emergency Departments (CONFIDENCE) Research Group. Children with Covid-19 in Pediatric Emergency Departments in Italy. N Engl J Med 2020 May 1.

16. Tagarro A, Epalza C, Santos M, et al. Screening and Severity of Coronavirus Disease 2019 (COVID-19) in Children in Madrid, Spain. JAMA Pediatr 2020 Apr 8.

17. Pathak EB, Salemi IL, Sobers N, Menard J, Hambleton IR. COVID19 in Children in the United States: Intensive Care Admissions, Estimated Total Infected, and Projected Numbers of Severe Pediatric Cases in 2020. J Public Health Manag Pract.2020 Apr 16.

18. Kam KQ, Yung CF, Cui L, et al. A Well Infant with Coronavirus Disease 2019 (COVID-19) with High Viral Load. Clin Infect Dis 2020 Feb 28.19 .

19. Su L, Ma X, Yu H, et al. The different clinical characteristics of corona virus disease cases between children and their families in China - the character of children with COVID-19. Emerg Microbes Infect 2020;9:707-13. 